\title{
Erratum to: Transitional Progenitors during Vertebrate Retinogenesis
}

\author{
Kangxin Jin $^{1}$ - Mengqing Xiang ${ }^{1,2}$
}

Published online: 8 August 2016

(C) Springer Science+Business Media New York 2016

Erratum to: Mol Neurobiol 2016

DOI: 10.1007/s12035-016-9899-x

The original paper of this article unfortunately contain errors. The author Mengqing Xiang was inadvertently omitted due to miscommunication. His contributions include important conceptual input, artworks, and funding supports.

The correct list of authors and affiliations as well as funding supports are here by published.

The authors apologize to the readers for these mistakes.

Acknowledgments The authors thank Drs. Courtni Newsome, MinZou, Shengguo Li, and Rashade A. H. Haynes II for critical readings and helpful comments on the manuscript. The authors apologize that so many great works in the field are not cited due to space limit and accessibility of full articles. This work was supported in part by the Fundamental Research Funds of the State Key Laboratory of Ophthalmology, Sun Yat-sen University, and the National Basic Research Program of China (973 Program, No. 2015CB964600) to MX.

The online version of the original article can be found at http://dx.doi. org/10.1007/s12035-016-9899-x.

Kangxin Jin

kxjin@yahoo.com

1 State Key Laboratory of Ophthalmology, Zhongshan Ophthalmic Center, Sun Yat-sen University, 54 South Xianlie Road, Guangzhou, Gongdong 510060, China

2 Center for Advanced Biotechnology and Medicine and Department of Pediatrics, Rutgers University-Robert Wood Johnson Medical School, 679 Hoes Lane West, Piscataway, New Jersey 08854, USA 